\title{
Partie 1: Anomalies des membres inférieurs
}

\author{
Sylvia Willi-Dähna, Hannes Manner ${ }^{\text {b }}$ \\ ${ }^{a}$ Oberärztin Kinder- und Jugendorthopädie; ${ }^{b}$ Leitender Arzt Kinder- und Jugendorthopädie, Schulthess Klinik Zürich
}

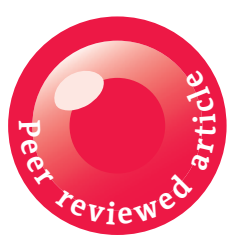

Dans l'article qui suit, nous allons décrire différentes anomalies essentielles et fréquentes de l'appareil locomoteur du membre inférieur et aborder les caractéristiques physiologiques et pathologiques. Les pathologies seront discutées en détail. Larticle doit aider à détecter précocement de potentiels problèmes afin que les petits patients puissent recevoir le traitement approprié tôt.

Connaître le développement normal de l'appareil locomoteur pendant la croissance est une condition sine qua non à l'évaluation correcte des résultats chez les enfants et les adolescents. En effet, l'angle de rotation et les axes des membres inférieurs, la forme des pieds et la marche évoluent au cours de la croissance de l'enfant.

\section{Anamnèse et examen clinique}

L'anamnèse précise et l'examen corporel sont essentiels. Les questions suivantes doivent être clarifiées:

- Qu'est-ce que les parents ont remarqué? Ces anomalies sont-elles survenues récemment ou bien ontelles été observées «depuis toujours»? Se sont-elles renforcées ou ont-elles régressé?

- L'enfant présente-t-il des douleurs?

- L'enfant présente-t-il des limitations fonctionnelles, par ex. lors de la marche?
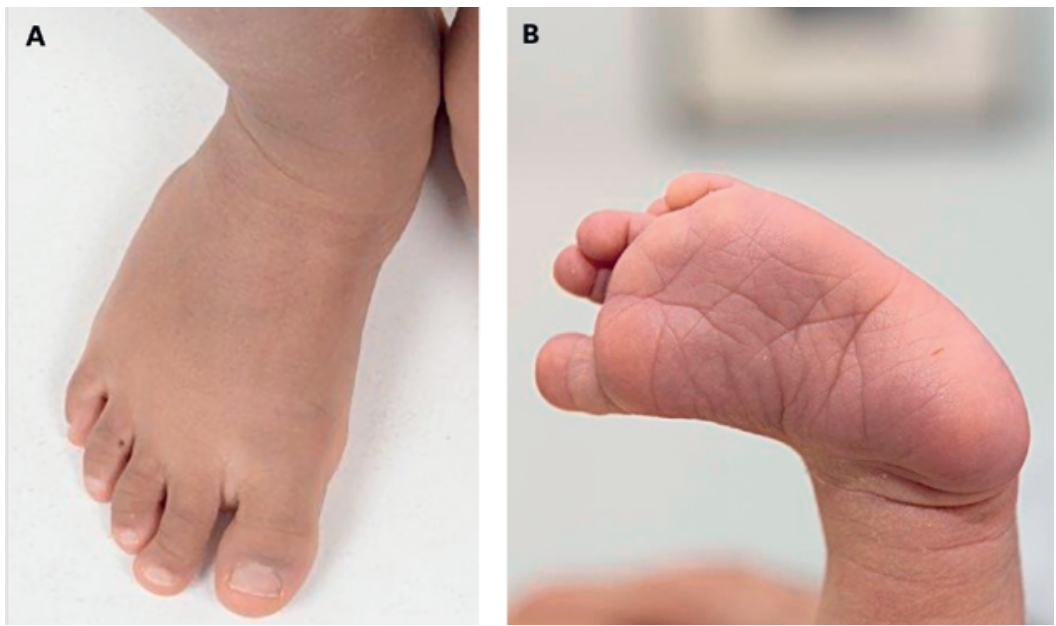

Figure 1: A: Léger pes adductus rigide (côté droit) chez un jeune enfant et B: Pes adductus positionnel pouvant être redressé chez le nourrisson.
- L'enfant est-il par ailleurs en bonne santé? (anomalies neurologiques?)

Lors de l'examen clinique, la marche (y compris l'axe des jambes), l'état articulaire précis, les éventuelles anomalies extérieures et la fonction musculaire sont examinés et évalués.

\section{Marche en rotation interne ou externe}

La marche en rotation interne marquée est une cause fréquente de consultation de l'orthopédiste pédiatrique. Les enfants trébuchent parfois sur leurs propres pieds; les plus jeunes enfants ne sont en revanche pratiquement jamais gênés.

\section{Pes adductus}

Lors de l'analyse de la marche en rotation interne, trois niveaux sont évalués. Dans un premier temps, la position du pied est examinée. S'agit-il d'un pes adductus (fig. 1)? Le pes adductus est la plupart du temps la conséquence d'un manque de place dans l'utérus. Le pronostic relatif à une amélioration spontanée de la situation est la plupart du temps très bon. En cas de pes adductus plus rigide, un redressement manuel ou un redressement au moyen d'un plâtre est nécessaire. Toutefois, plus l'enfant est âgé, plus ce type de traitement avec plâtre est laborieux et moins il a de chances de succès. Fort heureusement, des problèmes fonctionnels ne résultent pratiquement jamais de cette forme de pied. Dans de nombreux cas, seule une légère adduction fonctionnelle de l'avant-pied est présente, avant tout lors de la marche, et elle peut être redressée par l'examinateur. Elle disparaît la plupart du temps pendant la croissance et ne nécessite aucun traitement particulier. 


\section{Torsion tibiale}

La torsion du tibia doit ensuite être évaluée. Il s'agit de la rotation de la jambe entre l'articulation du genou et la fourche malléolaire, ou l'angle d'ouverture du pied. Souvent, chez les nourrissons, il n'y a pas encore de rotation tibiale externe ou de torsion interne du tibia (fig. 2). La torsion tibiale externe physiologique se développe jusqu'à l'âge de 6 ou 7 ans env. pour atteindre en moyenne $15-20^{\circ}$ par rapport à l'axe longitudinal du pied (angle d'ouverture du pied). Ainsi, une légère marche en rotation interne partant de la jambe est physiologique chez les jeunes enfants et ne nécessite pas d'autres mesures.

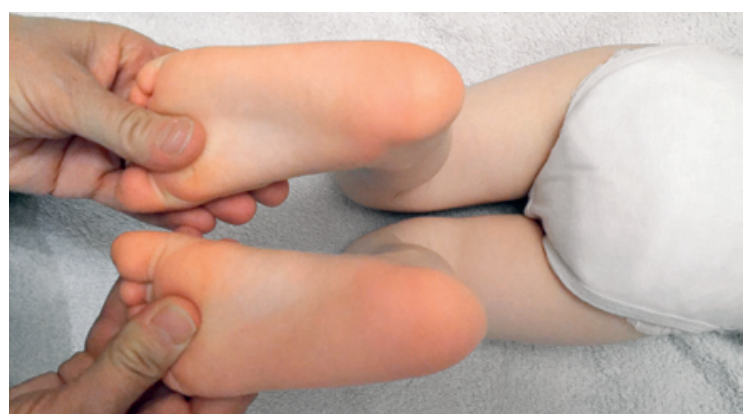

Figure 2: Torsion tibiale externe qui n'est pas encore développée chez un enfant de 2 ans.

\section{Antétorsion fémorale}

Chez l'enfant, la marche en rotation interne est le plus souvent induite par une antétorsion fémorale accrue. Il convient de noter que la détorsion spontanée du fémur est bien plus longue que la détorsion tibiale; elle dure jusqu'à la fin de la croissance. L'antétorsion fémorale est d'env. $30-50^{\circ}$ chez les nourrissons (fig. 3) et d'env. $15^{\circ}$ chez les adultes. La rotation interne de la hanche diminue donc durant la croissance. En cas de rotation de l'articulation de la hanche encore plus marquée du fait d'une antétorsion élevée, on observe un "kneeing-in" lors de la marche en ligne droite (marche en rotation fémorale interne); lors de la marche, les rotules ne regardent pas vers l'avant mais vers l'intérieur. Cela se manifeste alors aussi par un "toeing-in", ce qui se re-

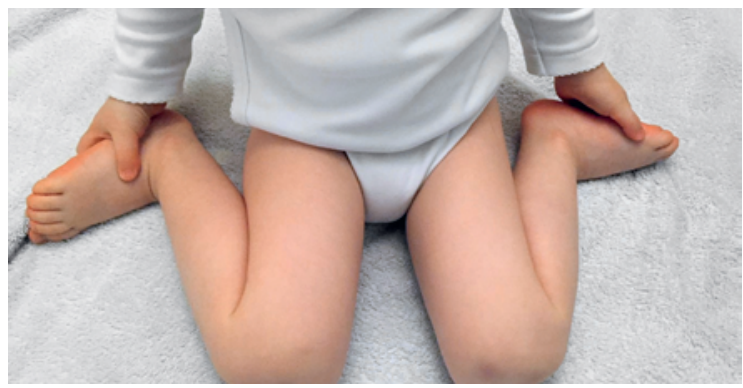

Figure 3: L'antétorsion fémorale renforcée induisant une rotation interne accrue de l'articulation de la hanche rend l'assise en position w (tailleur inversé) possible. marque le plus et inquiète le plus souvent les parents. Naturellement, un traitement par semelle orthopédique serait ici insensé, car la rotation interne du fémur n'est pas influencée par ce biais.

\section{La marche en rotation externe doit faire l'objet d'investigations}

Un jeune enfant qui marche en rotation interne présente donc une démarche physiologique naturelle. Des examens supplémentaires ne sont pas nécessaires. En revanche, si un enfant présente une marche en rotation externe prononcée, d'autres examens doivent être conduits. Une torsion tibiale externe accrue pourrait en être la cause, mais il pourrait également s'agir d'un pied plat valgus prononcé nécessitant un traitement.

\section{Quand une marche en rotation interne ou externe est-elle pathologique?}

Chez les adolescents, les rapports de rotation du fémur et du tibia ne changent plus considérablement. Chez certains adolescents, une trop forte antétorsion fémorale constitue une limitation fonctionnelle, surtout pendant le sport. Il n'est pas rare que les genoux se cognent l'un contre l'autre lors de la course rapide et que les personnes trébuchent. Des douleurs fonctionnelles dans la région des membres inférieurs sont parfois également rapportées. Le syndrome fémoro-rotulien, qui est classique chez les jeunes, peut trouver son origine dans une antétorsion fémorale accrue pathologique. L'antétorsion fémorale réduite ou absente (rétrotorsion fémorale) conduit à une marche en rotation externe (kneeing-out) et doit être considérée en tant que pré-arthrose de l'articulation de la hanche. En cas de douleurs inguinales, un examen approfondi des rapports de rotation de la hanche doit donc être conduit.

\section{Torsional malalignement}

Une forme particulière d'anomalie de torsion des membres inférieurs est le "torsional malalignement»: une antétorsion fémorale accrue, donc une rotation interne de la hanche trop élevée, survient en association avec une torsion externe de la jambe trop importante. Cette rotation "en sens opposé» des jambes et des cuisses induit un kneeing-in marqué avec un angle d'ouverture des pieds toutefois normal lors de la marche libre. Cette problématique est souvent méconnue. Seul un examen approfondi des jambes nues permet d'observer ce kneeing-in marqué. Lorsque l'on contraint le patient à marcher avec les rotules dirigées vers l'avant, la rotation tibiale externe pathologique marquée peut également être constatée sur la base des pieds fortement axés vers l'extérieur. Cette anomalie de torsion constitue souvent une limitation fonction- 
nelle, par ex. lors du déplacement à vélo ou de la course à pied. Des douleurs correspondantes se manifestent parfois dans la région du genou et des chevilles.

Les anomalies peuvent être idiopathiques, mais elles peuvent également trouver leur origine dans une fracture des membres inférieurs mal consolidée.

\section{Traitement des anomalies de torsion}

Dans la quasi-totalité des cas, la correction des anomalies de torsion symptomatiques n'est possible que par voie chirurgicale, au moyen d'opérations de dérotation du fémur ou du tibia. La correction doit naturellement être réalisée au niveau du défaut d'alignement. La physiothérapie et les semelles orthopédiques ne permettent malheureusement pas de modifier durablement ces anomalies de rotation.

\section{Défauts d'alignement de l'axe frontal des jambes: genu valgum (jambes en $\mathrm{X}$ ) et genu varum (jambes en 0 )}

Les axes frontaux des membres inférieurs changent pendant la croissance. A la naissance, on observe souvent des jambes en $\mathrm{O}$ (genu varum), qui sont accentuées par une rotation tibiale interne concomitante (fig. 4). Dès lors que l'enfant commence à marcher, l'axe des jambes se compense habituellement progressivement; en position debout lorsque les rotules sont centrées, les condyles fémoraux et les malléoles se touchent alors (fig. 5). On observe ensuite habituellement un développement des jambes légèrement en $\mathrm{X}$ (genu valgum, fig. 6) jusqu'à l'âge de 3-4 ans. Ces jambes en $\mathrm{X}$ se corrigent normalement à leur tour jusqu'à l'âge de la scolarité primaire.

Les jeunes enfants qui présentent un axe des jambes non pas en valgus mais en varus devraient faire l'objet d'examens supplémentaires ou du moins d'un suivi. En cas de marche précoce, on observe parfois tout d'abord un renforcement du varus au lieu d'une com-

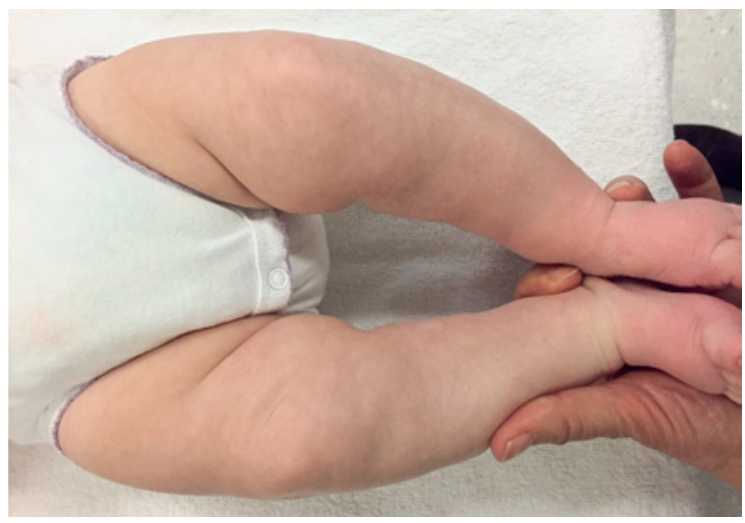

Figure 4: Genu varum chez le nourrisson; à cet âge, il est avant tout induit par la rotation interne physiologique. pensation de l'axe des jambes durant la croissance, mais habituellement, il se corrige rapidement. Cependant, le diagnostic différentiel doit également prendre en compte une possible maladie de Blount ou un potentiel rachitisme.

Un défaut d'alignement unilatéral nécessite également des examens supplémentaires. Une altération pathologique de la plaque épiphysaire (post-traumatique, tumeur, infection, malformation congénitale) peut en être la cause. En conséquence, ces patients devraient rapidement faire l'objet d'examens supplémentaires.

\section{Traitement des défauts d'alignement des axes}

Un défaut d'alignement des axes persistant peut non seulement causer une gêne fonctionnelle, mais il s'avère également défavorable sur le plan mécanique à moyen ou long terme (pré-arthrose, fig. 7). Il est possible de corriger un défaut d'alignement des jambes en $\mathrm{X}$ ou en $\mathrm{O}$ pendant la poussée de croissance pubertaire au moyen d'une petite intervention chirurgicale. Après l'analyse précise du défaut d'alignement sur la base d'un cliché radiographique de l'ensemble de la jambe, une petite plaque est implantée sur la plaque de croissance épiphysaire, ce qui freine la croissance au niveau de cette plaque du côté médial ou latéral (hémi-épiphysiodèse, souvent par eight plate, ou au moyen d'autres techniques telles que l'hémi-épiphysiodèse par forage ou agrafes) (fig. 8 et 9). De cette façon, l'axe des jambes se compense au cours de la croissance ultérieure. La correction des rapports axiaux réduit en particulier considérablement le risque de développer une gonarthrose.

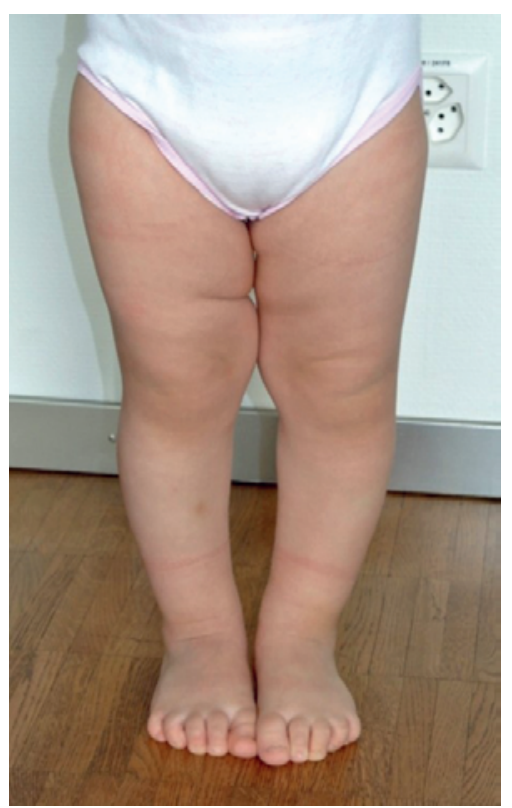

Figure 5: Axe des jambes compensé après le début de la marche, les condyles fémoraux et les malléoles se touchent. 


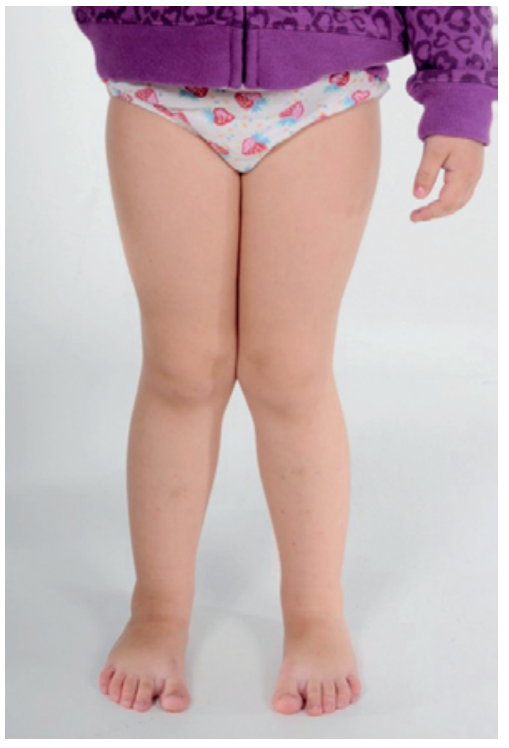

Figure 6: Genu valgum physiologique chez le jeune enfant: des examens supplémentaires ne sont pas nécessaires.

Dès lors que les plaques de croissance épiphysaires sont fermées à la fin de la croissance, une correction par «modulation de la croissance» n'est plus possible. L'axe des jambes peut alors encore uniquement être corrigé par des ostéotomies, ce qui représente une intervention considérablement plus lourde. C'est la raison pour laquelle les enfants qui présentent un genu valgum ou un genu varum devraient être présentés à un orthopédiste pédiatrique avant la poussée de croissance pubertaire, afin qu'une analyse axiale radiologique précise puisse être réalisée.

\section{Différences de longueur des jambes}

Les différences de longueur des jambes peuvent, en fonction de leur ampleur, conduire à une bascule du bassin. Elles conduisent également souvent à une inclinaison du sacrum et, de façon secondaire, à une scoliose de la colonne vertébrale. La bascule du bassin peut en outre aboutir à une couverture asymétrique défavorable des têtes fémorales. Les différences de longueur des jambes peuvent se renforcer pendant la croissance, et les causes potentielles de différence de longueur des jambes devraient dès lors également être évaluées lors de l'examen clinique. La plupart des différences de longueur des jambes sont idiopathiques. Lorsqu'elles sont inférieures à $1 \mathrm{~cm}$, elles ne posent généralement pas de problèmes et ne nécessitent pas de traitement. En cas d'hémihypotrophie, il y a non seulement une différence de longueur mais également de circonférence. Les luxations des hanches, la désaxation unilatérale ainsi que les malformations du pied et les malformations des membres inférieurs peuvent

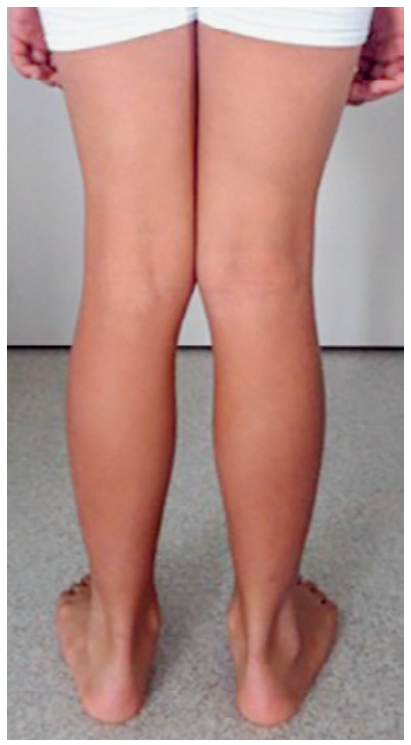

Figure 7: Genu valgum chez l'adolescent: un examen radiologique devrait être réalisé.

également conduire à l'apparition d'une jambe plus courte d'un côté que de l'autre.

Par ailleurs, les fractures dans la région des plaques épiphysaires peuvent entraîner un raccourcissement et un défaut d'alignement en raison de la fermeture précoce des plaques. En cas de fracture diaphysaire, une croissance osseuse accrue et donc une longueur excessive de la jambe peuvent survenir. En présence d'une hémi-hypertrophie, une malformation vasculaire peut être en cause. Il convient de distinguer ces différences de longueur des jambes réelles des différences fonctionnelles, par ex. induites par des contractures des articulations.

Lors de l'examen, la différence de longueur est compensée par de petites planches jusqu'à ce que la hanche soit droite en vue de déterminer l'ampleur de la différence.

\section{Traitement des différences de longueur des jambes}

Les différences de longueur des jambes de plus de 1-1,5 cm sont compensées du moins partiellement par des talonnettes. Pour les différences de plus de $2 \mathrm{~cm}$, des mesures chirurgicales sont également envisagées (ralentissement de la croissance, épiphysiodèse par perçage ou plaques, ostéotomies de raccourcissement, allongement du côté le plus court).

\section{Marche sur la pointe des pieds}

Chez un grand nombre de jeunes enfants, une marche sur la pointe des pieds ou l'avant-pied peut être observée, notamment lorsqu'ils sont excités. Ces enfants marchent parfois la majeure partie du temps sur 


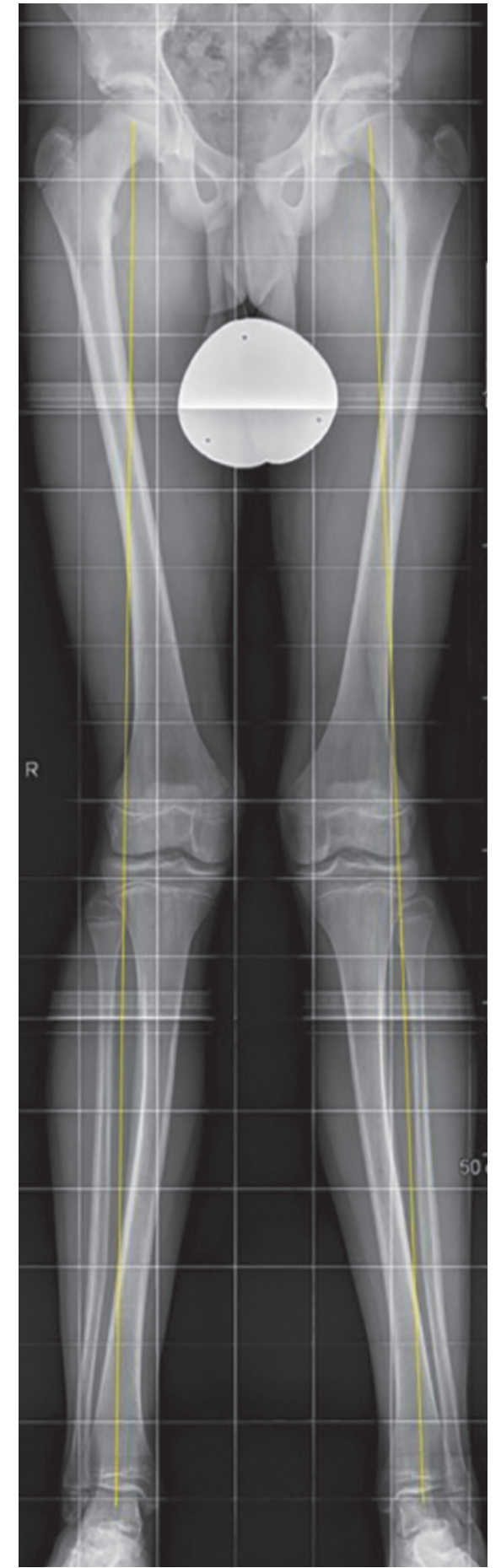

Figure 8: A la radiologie de ces jambes en $X$, on observe un décalage latéral de l'axe mécanique, soit une déviation axiale au sens d'une pré-arthrose, car le compartiment externe de l'articulation du genou est excessivement sollicité à long terme.

l'avant-pied, mais un contact normal talon-sol est toutefois également régulièrement observé. L'examen clinique évalue avant tout la longueur du muscle triceps sural ou l'extensibilité dorsale du pied au niveau de la cheville supérieure. Cet examen est conduit avec le

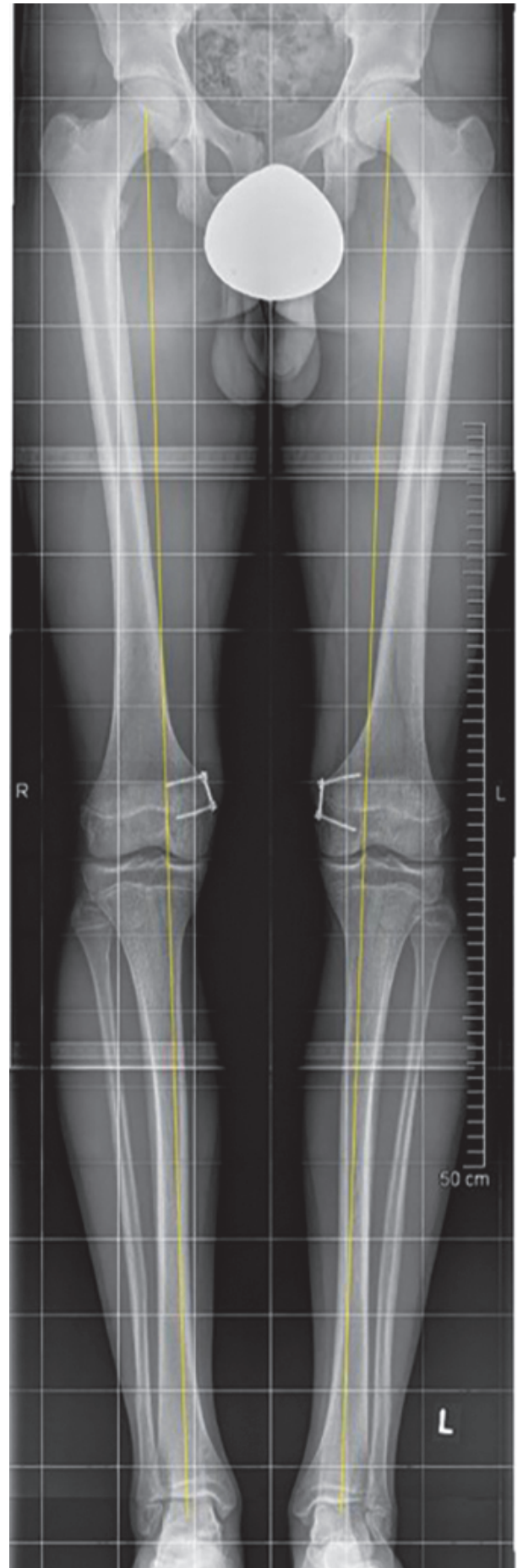

Figure 9: Au moyen d'une hémi-épiphysiodèse (modulation de la croissance), l'axe des jambes a pu être corrigé avec une petite intervention chirurgicale.

genou en extension, car le muscle se détend lorsque le genou est fléchi. L'extension dorsale devrait pouvoir être réalisée sans problème au-delà de la position neutre lorsque l'alignement du pied est corrigé (correction d'un potentiel pied plat valgus) (fig. 10). Si tel est le 


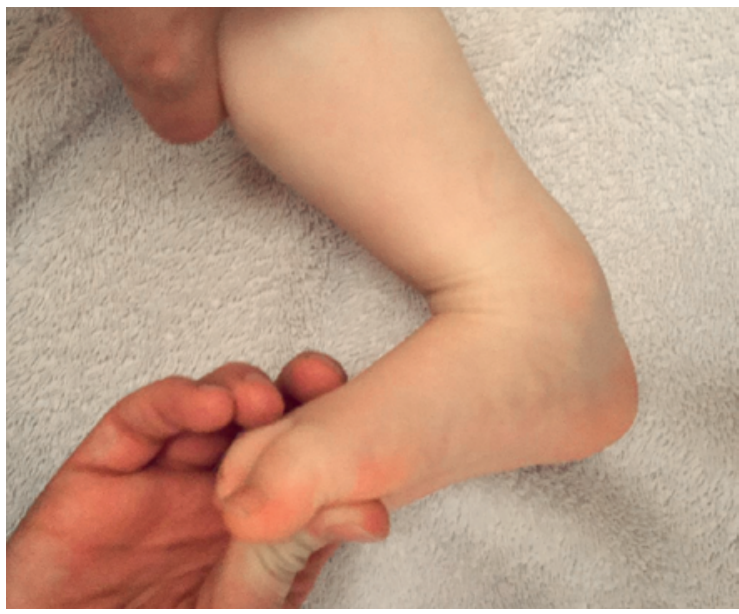

Figure 10: Chez un enfant sain sans raccourcissement musculaire, l'extension dorsale du pied au niveau de la cheville, au-delà de la position neutre, devrait pouvoir être réalisée sans problème.

cas, il s'agit alors le plus souvent de la cause la plus fréquente de la marche sur la pointe des pieds ou l'avantpied: la marche sur la pointe des pieds habituelle. Cette situation n'est pas problématique et le pronostic concernant le développement d'une marche normale est très bon. Dans les cas tenaces, avant tout chez les enfants plus âgés, il s'avère toutefois pertinent de mettre en œuvre un traitement physiothérapeutique pour l'entraînement à la marche ou de prescrire des semelles pyramidales et de réaliser régulièrement des exercices d'étirement des triceps suraux.

Une marche sur la pointe des pieds peut toutefois également être le signe d'une véritable pathologie. Lorsqu'une extensibilité dorsale réduite, c.-à-d. raccourcissement du triceps, est constatée à l'examen clinique, ce raccourcissement peut être congénital ou
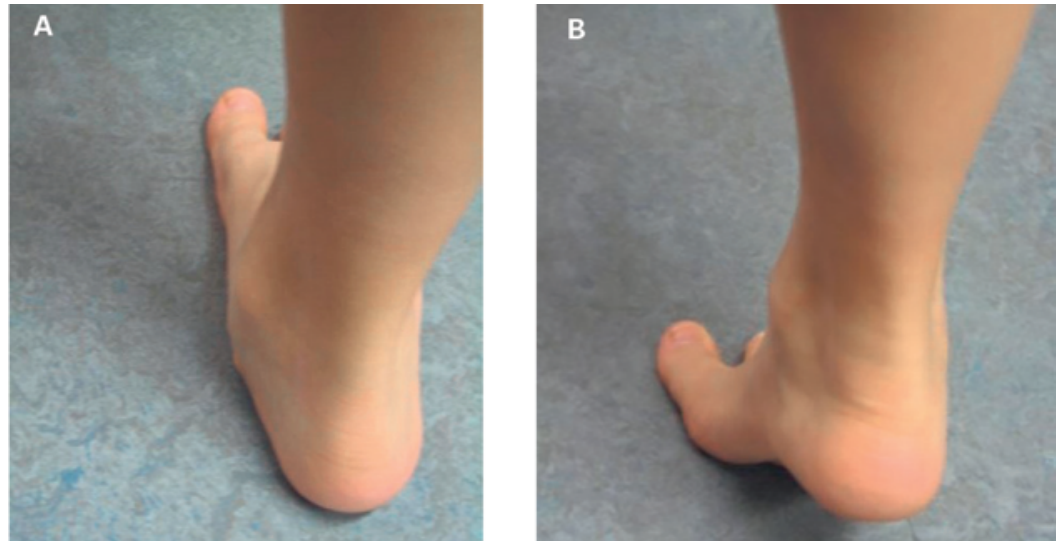

Figure 11: En cas de pied plat valgus flexible, on observe un redressement du pied en position debout sur la pointe des pieds (le talon se tourne vers l'intérieur et la voûte plantaire se redresse). acquis. Les raccourcissements observés peuvent être post-traumatiques, positionnels ou faire suite à une immobilisation par un plâtre. Ils peuvent également être secondaires à une différence de longueur des jambes (compensation fonctionnelle de la différence) ou apparaître dans le cadre d'un pied bot.

Il convient de ne pas manquer une cause neurogène de la marche sur la pointe des pieds. Un examen neurologique du moins sommaire devrait être réalisé chez tous les enfants qui présentent une marche sur la pointe des pieds. En cas de suspicion d'une composante neurogène, l'enfant doit également être présenté à un neuropédiatre.

\section{Traitement du pied équin}

L'approche thérapeutique est définie en fonction de l'ampleur du raccourcissement musculaire ou du pied équin et de la cause sous-jacente. L'objectif de toutes les formes de traitement est l'atteinte d'une position plantigrade du pied, ou, encore mieux, d'une bonne capacité d'extension dorsale au niveau de la cheville supérieure. En cas de raccourcissement minime du muscle, un traitement physiothérapeutique, un redressement au moyen d'un plâtre ou une prise en charge au moyen de semelles pyramidales suffit. En cas de fort raccourcissement, un allongement du tendon d'Achille ou un allongement aponévrotique du muscle doivent également être discutés. L'éventualité d'une composante osseuse doit également être prise en compte et, le cas échéant, être corrigée chirurgicalement. En cas de cause neurogène, une prise en charge avec orthèse est la plupart du temps nécessaire afin de prévenir une progression ou de réduire le risque de récidive après un autre traitement.

\section{Le pied plat valgus chez l'enfant}

Chez l'enfant, le pied plat valgus, qui va parfois de pair avec une usure inégale des chaussures, est une cause fréquente d'inquiétude des parents et grands-parents, qui demandent alors s'il ne serait pas pertinent d'utiliser des semelles correctrices. Mais dans quelle mesure ce traitement est-il pertinent?

La majorité des jeunes enfants présentent un pied plat valgus résultant d'une laxicité encore importante ou d'un coussinet adipeux plantaire important. Ce pied plat valgus est en outre renforcé par l'axe en valgus des jambes, qui est physiologique pendant la petite enfance. En règle générale, la forme du pied se normalise en grande partie jusqu'à l'âge de la scolarité primaire. Des études conduites à la fin des années 1980/au début des années 1990 ont montré que la forme du pied ne pouvait pas être influencée de façon durable par des se- 
melles. Un traitement n'est pas nécessaire dès lors que le pied est flexible à l'examen clinique, qu'aucun épaississement en tant que manifestation d'une véritable sollicitation anormale du pied n'est présent, que le pied se redresse en position sur la pointe des pieds (fig. 11) et que l'anamnèse n'a pas indiqué de douleurs.

Il convient de ne pas manquer d'éventuelles coalitions (jointures pathologiques congénitales entre deux os du pied) dans la région du pied en tant que cause de potentielles douleurs et d'un pied plat valgus s'accentuant de façon secondaire au cours de l'évolution. Les troubles surviennent le plus souvent à l'adolescence, souvent à la suite d'une entorse dans le cadre de laquelle la coalition subit un traumatisme. En cas de coalitions talo-naviculaires, plus fréquentes, les douleurs se projettent sur le sinus du tarse ou l'articulation de Chopart (localisation antéro-externe par rapport à la cheville); en cas de coalitions sous-taliennes, plus rares, elles se projettent sur le côté intérieur de l'articulation inférieure de la cheville, sous la malléole interne. Ici, il n'est pas rare d'observer la protubérance pathognomonique du sustentaculum tali. Sur le plan clinique, un pied plat valgus s'accentuant et se montrant très rigide est souvent observé. En position debout sur la pointe des pieds, le pied ne se redresse pas ou très faiblement, le talon reste en valgus. La mobilité de l'articulation de la cheville inférieure ou de l'articulation de Chopart est réduite ou absente, avec des douleurs en cas de mouvement forcé.

Les coalitions sont malheureusement souvent manquées. Cela conduit fréquemment à des douleurs durant de nombreuses années. En cas de douleurs de l'arrière-pied persistantes ou de pied plat valgus s'in- tensifiant ou rigide, le patient doit donc passer des examens supplémentaires pour rechercher une éventuelle coalition.

En cas de suspicion clinique, le diagnostic est posé sur la base de la radiographie (cliché oblique du pied) et, notamment en cas de coalitions fibreuses, sur la base de l'IRM.

\section{Traitement du pied plat valgus}

Si des troubles surviennent avec le temps en cas de pied plat valgus chez l'enfant, selon l'ampleur de la déformation du pied, un traitement par semelles plantaires sur mesure à rembourrage souple est en premier lieu initié. Le traitement a pour objectif de décharger le pied et d'obtenir une absence de douleurs.

En cas de pied plat valgus très marqué et de symptômes persistants malgré les mesures conservatrices, un redressement chirurgical peut également être discuté.

En présence d'une coalition, le traitement par semelles correctrices n'est pas efficace, car le pied n'est pas flexible. Souvent, les semelles ne sont pas supportées. En cas de douleurs, l'indication d'une résection de la coalition ou d'une arthrodèse de l'articulation inférieure de la cheville est posée.

\section{À noter}

La partie 2 de cet article paraîtra dans le prochain numéro de Primary and Hospital Care sous le titre "Asymétries du tronc et scoliose». 